\title{
Coherence Effects in L-Band Active and Passive Remote Sensing of Quasi-Periodic Corn Canopies
}

\author{
Cuneyt Utku ${ }^{1}$, Roger H. Lang ${ }^{2}$ \\ ${ }^{1}$ Goddard Earth Sciences \& Technology Center, Goddard Space Flight Center, Greenbelt, MD 20771, USA \\ e-mail: Cuneyt.Utku@nasa.gov \\ ${ }^{2}$ The George Washington University, Washington, D.C. 20052, USA \\ e-mail: lang@gwu.edu
}

\begin{abstract}
Due to their highly random nature, vegetation canopies can be modeled using the incoherent transport theory for active and passive remote sensing applications. Agricultural vegetation canopies however are generally more structured than natural vegetation. The inherent row structure in agricultural canopies induces coherence effects disregarded by the transport theory. The objective of this study is to demonstrate, via Monte-Carlo simulations, these coherence effects on L-band scattering and thermal emission from corn canopies consisting of only stalks.
\end{abstract}

\section{Introduction}

In this study, we demonstrate via Monte-Carlo (MC) simulations, the effect coherence induced by vegetation canopy row structure on active and passive remote sensing of the canopy. For this purpose, a simple defoliated corn canopy consisting of only stalks is considered. In order to imitate the canopy row structure, the stalks are distributed with a quasi-periodic distribution (QPD) where they are first placed on a periodic lattice and later given random displacements from their positions on the periodic lattice. Monte-Carlo simulations are performed for the mean backscattering coefficient and brightness temperature at L-band for sensors looking along the row direction and perpendicular to the row direction to observe the effect of coherence. Results are also compared with those from uniform canopies with stalks distributed uniformly throughout the canopy. Discussion on the implications of the effect of coherence is given at the end.

\section{Quasi-Periodic Canopy Model}

The quasi-periodic canopy model consists of stalks (modeled as finite length dielectric cylinders) randomly placed with a quasi-periodic distribution over a perfect electric conductor (PEC) plane. For simplicity, the leaves and cobs are assumed to be removed from the stalks. The QPD can be regarded as a non-ideal periodic structure. It is obtained from the periodic lattice of the cylinders by randomly displacing the cylinders from their ideal positions on the periodic lattice. The cylinders are displaced within a bounding region according to a prescribed distribution with zero mean. In this manner, all cylinders are identically distributed about their ideal positions on the lattice. Note that the periodic canopy can be obtained from the quasi-periodic canopy by choosing a Dirac delta distribution. A top view of the quasi-periodic canopy with circular bounding regions of radius $b$, is depicted in Fig.1. In the figure, $\Delta_{x}$ and $\Delta_{y}$ correspond to the row spacing and the in-row cylinder spacing respectively. The canopy composed of finite length cylinders over a PEC plane is depicted in Fig.2.

\section{Analysis}

For the active problem, we consider a unit amplitude plane wave with wavenumber, $k_{o}$, incident on the canopy in the direction defined by the angle pair $\left(\theta_{i}, \phi_{i}\right)$ where the angle $\theta_{i}$ is measured from the $z$-axis perpendicular to the PEC plane and the azimuth angle $\phi_{i}$ is measured from $x$-axis in the row direction. The corresponding incidence vector $\boldsymbol{k}$ is shown in Fig 2. The mean bistatic cross section of the canopy is defined as:

$$
\left\langle\sigma_{p q}^{\circ}\left(\boldsymbol{k}^{\prime}, \boldsymbol{k}\right)\right\rangle=\lim _{r \rightarrow \infty} \frac{\left\langle\left|E_{s, q}\left(\boldsymbol{k}^{\prime}, \boldsymbol{k}\right)\right|^{2}\right\rangle}{A\left|E_{o, p}(\boldsymbol{k})\right|^{2}}=\lim _{r \rightarrow \infty} \frac{1}{A\left|E_{o, p}(\boldsymbol{k})\right|^{2}}\left\{\left|\left\langle E_{s, q}\left(\boldsymbol{k}^{\prime}, \boldsymbol{k}\right)\right\rangle\right|^{2}+\left\langle\left|E_{s f, q}\left(\boldsymbol{k}^{\prime}, \boldsymbol{k}\right)\right|^{2}\right\rangle\right\}
$$




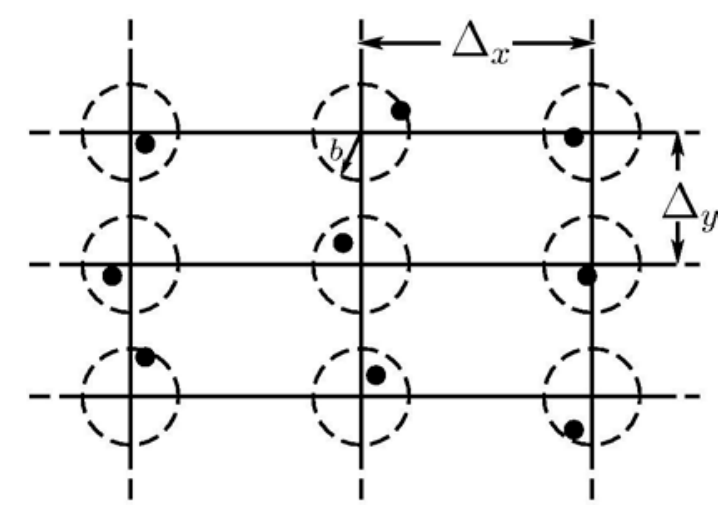

Figure 1. Top view of a quasi-periodic canopy.

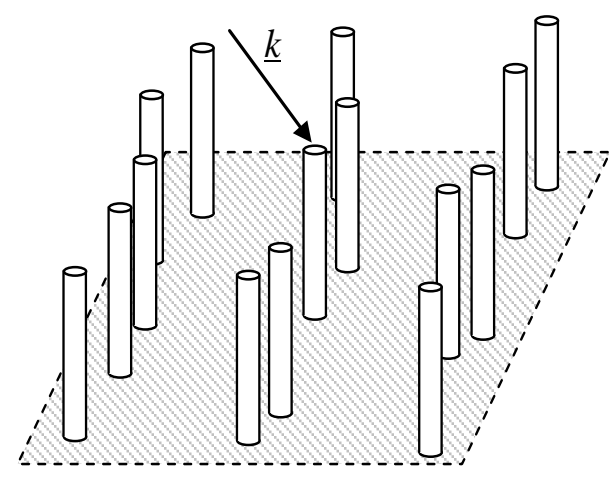

Figure 2. Model for corn canopy composed of finite length dielectric cylinders over a PEC plane.

In the above, $r$ is the distance of observation from the canopy, $E_{o, p}$ and $E_{s, q}$ are the $p$-polarized incident and $q$ polarized scattered field amplitudes respectively $(p, q \in\{h, v\}), A$ is the area of the canopy and \langle\rangle denotes the average over cylinder configurations. The term $E_{s f, q}$ is the $q$-polarized fluctuating part of the scattered field defined as $E_{s f, q}=E_{s, q}-\left\langle E_{s, q}\right\rangle$. The quantity of interest in this study is the mean backscattering coefficient obtained from (1) with $\boldsymbol{k}^{\prime}=-\boldsymbol{k}$. Considering the mean scattered field, $\left\langle E_{s, q}\right\rangle$, it is clear that a QPD canopy of infinite extent is invariant under translations by multiples of $\Delta_{x}$ or $\Delta_{y}$ in the $x$ and $y$ directions respectively. This will lead to space harmonics for the mean scattered field determined by the angles

$$
\cos \theta_{m n}=\left[1-\left(\sin \theta_{i} \cos \phi_{i}+\frac{2 \pi m}{k_{o} \Delta_{x}}\right)^{2}-\left(\sin \theta_{i} \sin \phi_{i}+\frac{2 \pi n}{k_{o} \Delta_{y}}\right)^{2}\right]^{1 / 2}
$$

where $m, n \in \mathbb{Z}$ i.e. integer. For a canopy of finite extent, the space harmonics will persist but with finite angular width inversely related to the canopy extent.

For the passive problem, the emissivity of the canopy is given by the generalized Kirchhoff law i.e. the emissivity of the canopy is equal to its absorbtivity under thermal equilibrium. Under thermal equilibrium the active and passive problems are related as follows: emission in the $\left(\theta_{i}, \phi_{i}\right)$ direction is obtained from the absorption by the stalks due to a unit amplitude plane wave incident on the canopy in the $\left(\theta_{i}, \phi_{i}\right)$ direction. Precisely, the $p$-polarized emissivity of the canopy is given by:

$$
\left\langle e_{p}\left(\theta_{i}, \phi_{i}\right)\right\rangle=\left\langle\sigma_{a p}^{\circ}\left(\theta_{i}, \phi_{i}\right)\right\rangle=1-\frac{1}{4 \pi} \int_{2 \pi}\left[\left\langle\sigma_{p p}^{\circ}\left(\theta, \phi, \theta_{i}, \phi_{i}\right)\right\rangle+\left\langle\sigma_{q p}^{\circ}\left(\theta, \phi, \theta_{i}, \phi_{i}\right)\right\rangle\right] d \Omega
$$

where $\sigma_{a p}^{\circ}\left(\theta_{i}, \phi_{i}\right)$ is the absorbtivity of the canopy due to the unit amplitude $p$-polarized plane wave incident in the $\left(\theta_{i}, \phi_{i}\right)$ direction and $d \Omega=\sin \theta d \theta d \phi$ is the differential solid angle. The above expression is commonly known as Peake's principle [1]. Note that since the cylinders (stalks) are placed over a PEC plane, absorption (hence emission) will correspond only to power absorbed (emitted) by the cylinders.

In order to compute the scattered and absorbed powers, the fields on the surface of the cylinders must be known. For this purpose, using a cylindrical harmonic expansion, a set of self-consistent (Foldy-Lax) equations is constructed for the coefficients of the surface fields of the cylinders. We emphasize that all multiple scattering is taken into account in this approach. The solution to the self-consistent equations requires a large matrix inversion that is performed using a bi-conjugate gradient method in conjunction with an FFT algorithm to speed up matrixvector multiplication. Details of the implementation can be found in [2] and [3]. Once the surface fields are obtained, the backscattering coefficient and the emissivity can be found using (1) and (3) respectively. In this study, emissivity is alternatively obtained from the power absorbed by the cylinders via a surface integral of the Poynting vector on the surface of the cylinders [3].

The averaging of the backscattering coefficient and emissivity is performed via MC averaging. Each realization of the simulation procedure consists of (i) generating a sample quasi-periodic canopy, (ii) solving for the coefficients of the cylinder surface fields (iii) computing the backscattering coefficient and the absorption cross section of the canopy. Mean backscattering and emissivity are obtained by averaging over all MC realizations. 


\section{Simulation Results and Discussion}

In this section, simulation results are given for the mean backscattering coefficients and mean emissions for two quasi-periodic corn canopies. Simulations are performed for both parallel-to-row and perpendicular-to-row look directions. In addition, we associate a uniform canopy with each quasi-periodic canopy such that the canopy has the same density as its associated quasi-periodic canopy. The stalks in the canopy have a uniform identical and independent distribution over the canopy area, $A$. Simulations are performed for these uniform canopies to facilitate comparison with their associated quasi-periodic canopies.

The simulations are performed at $1.5 \mathrm{GHz}$ for vertical polarization. Each MC simulation is the result of averaging over 50 realizations. The dielectric cylinders representing the stalks have radius $a=1 \mathrm{~cm}$, length $L=1 \mathrm{~m}$ and relative permittivity $\varepsilon_{r}=15+i 5$ and are identical for all canopies. A total of 512 cylinders are used in each canopy. Other relevant canopy parameters are given in Table 1.

Table 1. Canopy Parameters for MC Simulations

\begin{tabular}{|c|c|c|}
\hline & Canopy-1 & Canopy-2 \\
\hline Stalk Density $\# / \mathrm{m}^{2}$ & 8 & 6.7 \\
\hline Row spacing $(\mathrm{m}), \Delta_{x}$ & 0.50 & 0.75 \\
\hline Average stalk spacing $(\mathrm{m}), \Delta_{y}$ & 0.25 & 0.20 \\
\hline Bounding region radius, $b(\mathrm{~m})$ & 0.10 & 0.08 \\
\hline
\end{tabular}

Simulations results of mean backscattering coefficient vs. incidence angle for Canopy-1 and Canopy-2 are given in Fig. 3a and Fig. 3b respectively. In both figures three curves are plotted. The curves denoted by QPD( $\|)$ and $\mathrm{QPD}(\perp)$ correspond to quasi-periodic canopy results with parallel-to-row and perpendicular-to-row looks at the canopy respectively. The curve denoted by UIID corresponds to results of the associated uniform canopy. It is seen from Fig. 3a that for Canopy- 1 the three curves are within $2 \mathrm{~dB}$ of each other. For Canopy-2 however, above $30^{\circ}$ incidence there is about $5 \mathrm{~dB}$ difference between backscattering coefficients for the perpendicular look at the QPD canopy and the uniform canopy. The parallel look at the QPD canopy, on the other hand, has a conspicuous peak at $30^{\circ}$ incidence angle. This peak corresponds to a space harmonic occurring in the backscattering direction as can be computed from (2) with values from Table 1 corresponding to Canopy-2.

Simulation results of mean brightness temperature vs. incidence (observation) angle for Canopy-1 and Canopy-2 are given in Fig. 4a and Fig. 4b respectively. The curves in Fig. 4a show that the brightness temperatures for the parallel and perpendicular looks at Canopy- 1 are very similar over the complete angle range with a maximum difference of about $10 \mathrm{~K}$. The brightness temperatures for both parallel and perpendicular looks at the QPD canopy differ from those of the uniform canopy with a maximum difference at $\theta_{i}=40^{\circ}$ of about $36 \mathrm{~K}$ between the parallel look and the uniform canopy and a maximum difference of about $26 \mathrm{~K}$ between the perpendicular look and the
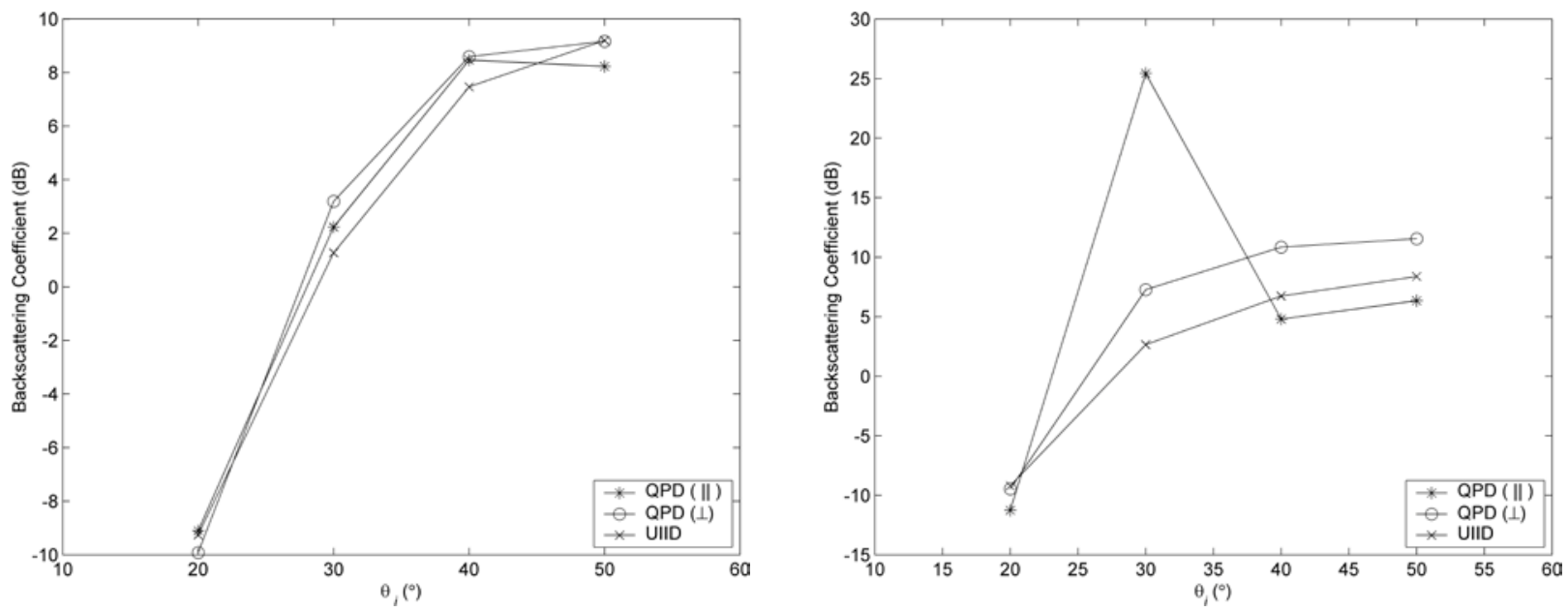

Figure 3. MC simulations of backscattering coefficient vs. incidence angle: (a) results for Canopy-1 (b) results for Canopy-2. 

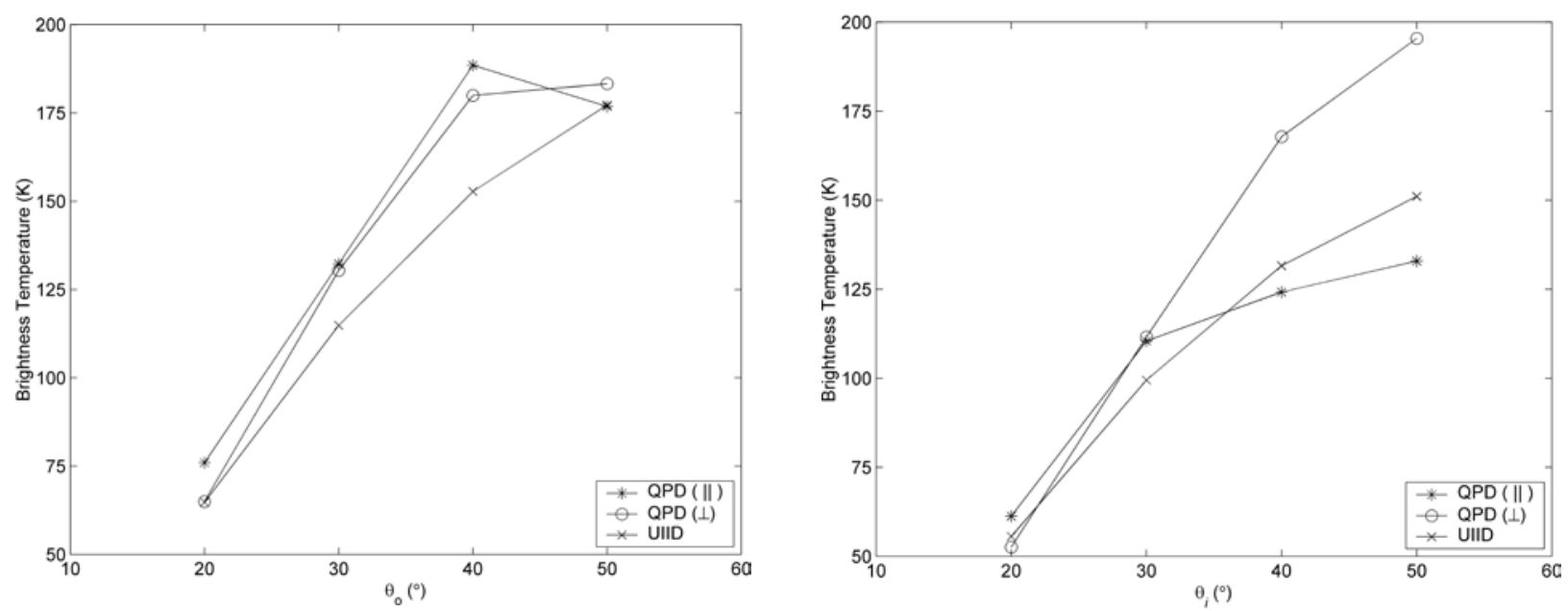

Figure 4. MC simulations of brightness temperature vs. observation angle: (a) results for Canopy-1 (b) results for Canopy-2.

uniform canopy. For Canopy-2, Fig. 4b shows that the brightness temperatures for the parallel and perpendicular looks are close up to $\theta_{i}=30^{\circ}$. As the incidence angle is increased, a gradual increasing in difference is observed. The maximum difference in the brightness temperatures between these looks is approximately $63 \mathrm{~K}$ at $\theta_{i}=50^{\circ}$. It is also observed that the brightness temperature of the uniform canopy is close to that of the QPD canopy up to $\theta_{i}=$ $30^{\circ}$ and at higher angles falls between the curves of the parallel and perpendicular looks at the QPD canopy.

\section{Conclusion}

Simulation results confirm the effect of coherence in both active and passive remote sensing of quasi-periodic canopies. In the active problem, backscattering can be substantially influenced by coherence if a space harmonic coincides with the backscattering direction in which case the uniform canopy assumption fails. This implies that for such a configuration, the incoherent transport theory analysis, where a uniform canopy is generally assumed, will not be adequate. An analysis using transport theory with non-uniform canopy assumption is given in [4] where it is also reported that taking coherence into account yields better results.

In the passive problem, the effect of coherence is generally visible at higher observation angles. The results also render transport theory analysis of emission from such quasi-periodic questionable. It is also worth noting, a direct relation between emission and space harmonics is not deduced, nevertheless they enjoy an indirect relationship through Peake's principle given by (3).

The above results are obtained for defoliated corn canopies. In a real canopy, the leaves will attenuate multiple scattering between stalks and hence decrease the effect of coherence and consequently the dependence of emission on the row structure. The incorporation of the leaves into the current model requires further study.

\section{References}

[1] W. H. Peake, "Interaction of electromagnetic waves with some natural surfaces," IEEE Trans. Antennas Propagat., vol. 7, pp. S324-S329, 1959.

[2] L. Tsang, K. H. Ding, G. Zhang, C. C. Hsu and J. A. Kong, "Backscattering enhancement and clustering effects of randomly distributed dielectric cylinders overlying a dieletric half space based on Monte-Carlo simulations," IEEE Trans. Antennas Propagat., vol. 43, pp. 488-499, May 1995.

[3] C. Utku, "The effective scatterer approximation in mean field modeling of random media with application to vegetation canopies,” Ph.D. dissertation, Dept. Elect. Eng., George Washington Univ., Washington, D.C., 2007.

[4] M.W. Whitt and F.T. Ulaby, "Radar response of periodic vegetation canopies," International Journal of Remote Sensing, vol. 15, no. 9, pp. 1813-1848, 1994. 